\title{
A Dense Pressure Sensitive Bedsheet Design for Unobtrusive Sleep Posture Monitoring
}

\author{
Jason J. Liu ${ }^{1}$, Wenyao $\mathrm{Xu}^{1}$, Ming-Chun Huang ${ }^{1}$, Nabil Alshurafa ${ }^{1}$, \\ Majid Sarrafzadeh $^{1}$, Nitin Raut ${ }^{2}$, Behrooz Yadegar ${ }^{2}$ \\ 1. Wireless Health Institute, Department of Computer Science, \\ University of California, Los Angeles, California, 90095 \\ 2. Medisens Wireless Inc., Santa Clara, California, 95051
}

\begin{abstract}
Sleep plays a pivotal role in the quality of life, and sleep posture is related to many medical conditions such as sleep apnea. In this paper, we design a dense pressuresensitive bedsheet for sleep posture monitoring. In contrast to existing techniques, our bedsheet system offers a completely unobtrusive method using comfortable textile sensors. Based on high-resolution pressure distributions from the bedsheet, we develop a novel framework for pressure image analysis to monitor sleep postures, including a set of geometrical features for sleep posture characterization and three sparse classifiers for posture recognition. We run a pilot study and evaluate the performance of our methods with 14 subjects to analyze 6 common postures. The experimental results show that our proposed method enables reliable sleep posture recognition and offers better overall performance than state-of-the-art methods, achieving up to $83.0 \%$ precision and $83.2 \%$ recall on average.
\end{abstract}

Keywords-Sleep Posture, Bedsheet, Sparse Classifier, Pressure Image Analysis.

\section{INTRODUCTION}

As the best way to heal the human body and recover energy, sleep has many implications on our overall health. It has been widely used as a diagnostic indicator in diverse medical applications. For example, sleep stage is a proven biometric in diagnosing cardiovascular disease, diabetes and obesity [1]. Sleep difficulty is associated with psychiatric disorders such as depression, alcoholism and bipolar disorder [2].

Among the indicators of determining sleep quality (such as sleep stage and sleep difficulty), sleep posture is one of the most important factors and is heavily used in performing medical diagnosis.

There are many medical conditions affected by sleep posture. One of the most common is sleep apnea. There have been several research works on sleep apnea analysis with sleep postures in recent years. Lee et al. reported that lateral (lying on side) postures can reduce sleep disorders for mild and moderate sleep apnea patients [3]. Ambrogio et al. discovered the relationship between sleep postures and chronic respiratory insufficiency, which leads directly to sleep apnea [4]. Oksenberg and Silverberg investigated breathing disorder and sleep postures [5]. They suggested that patients with obstructive sleep apnea should avoid sleep in the supine (lying on back) position.
Another medical application that is affected by sleep posture is the recovery from serious operative procedures. It has been shown that sleep quality affects the recovery times of patients [6]. More specifically, one of the main problems for post-surgical patients and elderly patients is formation of pressure ulcers [7]. Pressure ulcers, or bedsores, are localized injury to body tissue, usually near the bone, resulting from low blood circulation and lack of movement. Hospital staff need to be attentive to subjects that are more susceptible to this condition, and take action to relieve pressure on the highly sensitive locations by changing their sleep postures. Current best practices in nursing involve moving patients every several hours. However, there is no guarantee that patients remain in one posture in the meantime.

Given these applications, autonomously monitoring patients during recovery is desired, especially when pressure ulcers can develop very quickly. The goal of any medical system that prevents the formation of pressure ulcers requires the analysis of sleeping postures, as well as notifications of susceptible and impending pressure points on the patient's body. Other health issues associated with sleep postures include snoring, night sweats, and narcolepsy. Therefore, there is indeed a need for automatic sleep posture monitoring.

To date, researchers have proposed different ways to monitor sleep posture automatically. Video cameras and microphones have been used previously to study sleep posture patterns. For example, Nakajima et al. [8] prototyped a system based on visual signals to analyze sleep posture changes. However, the drawbacks of using video involve lighting issues. Low light levels at night adds noise to the images, and even when near-infrared cameras are used [9] the images still produced non-uniformity and artifacts. Furthermore, video and voice taping raise serious privacy concerns for users.

Inertial sensors, including accelerometers, gyroscopes and magnetometers, are another applied technique used to monitor sleep. Sadeh and Acebo attached several tri-axial accelerometers on limbs of people to monitor the sleep via actigraph [10]. Kishimoto et al. deployed 14 wearable motion sensors on users at home for remote sleep posture analysis 
[11]. The main downside to this technique is that sensors have to be attached to body which can be uncomfortable or burdensome to the users.

Alternatively, dispersed pressure sensors deployed in the mattress can record when changes in body posture occur. This method is unobtrusive and does not interfere in the comfort of users. Also it is a stable medium that is not affected by changes in the environment. Hoque et al. facilitated a mattress with wireless-powered accelerometers to record the movement activity [12]. Jones et al. developed a bedsheet system with 24 pressure sensors [13]. However, the focus was on detecting posture change rather than recognizing body posture.

In this paper, we focus on sleep posture analysis using pressure sensors. We employ a dense pressure sensitive textile bedsheet and apply pressure image analysis for sleep posture recognition. Our contribution in this work is threefold. First, we propose a framework for automatic sleep posture analysis based on a dense pressure sensitive bedsheet prototype $(64 \times 128$ sensors $)$ with e-textile material. We would like to argue that pressure image analysis is more challenging than visual image due to the stronger self-occlusion (examples will be shown in the following sections). Second, we define and discuss a set of geometric features from pressure images for posture analysis. These features are effective to not only distinguish different postures but characterize each posture with physical meanings (the details about geometric features will be elaborated in Section III). Third, we develop three heuristics based on sparse representation to classify sleep postures. We evaluate our proposed methods with 14 subjects for 6 common sleep postures.

The proposed method exhibits better performance in terms of accuracy and robustness than state-of-the-art methods. Since people do not usually change sleep postures often, we focus only on steady state sleep postures. Although there may be information from previous postures and during the transitions from one posture to another, we do not consider this in the current scope of this paper.

The remaining part of the paper is organized as follows. Section II describes the overall design of this monitoring system that incorporates a pressure sensitive bedsheet. Section III describes the algorithmic process of sleep posture recognition by extracting pressure image features and classification using the theory of the Sparse Classifiers. Experimental set up and results are given in Section IV. Finally, future work and conclusion are discussed in Section V.

\section{Bedsheet Design}

In this section, we present the design of the bedsheet system. The goal of this specialized bedsheet is to record the pressure distribution of the body while sleeping and then perform data analysis for medical applications. For instance, when a patient has had recent surgery around the left hip, limited pressure should be applied on that area and a leftlying posture should not be allowed. When the bedsheet system detects that the patient is lying on his left side for a period of time, the patient or caregivers can receive an alert to change the posture. Accordingly, the bedsheet system is designed with the following consideration:

- High-resolution: The bedsheet should offer high resolution for pressure sensing. Given enough resolution, it is possible to quantify the applied pressure on body parts and enable high accuracy medical diagnosis.

- Comfort: The user should feel comfortable lying on the sheet. Also, it should be easy to deploy in the home or hospital.

- Low-cost: For widespread use, the cost for the bedsheet implementation should be low and affordable for most people.

There are some existing sensor products [14], [15] that comprise of many piezo-electrical pressure sensors. However, none of them meets the above design criterions for wide applications.

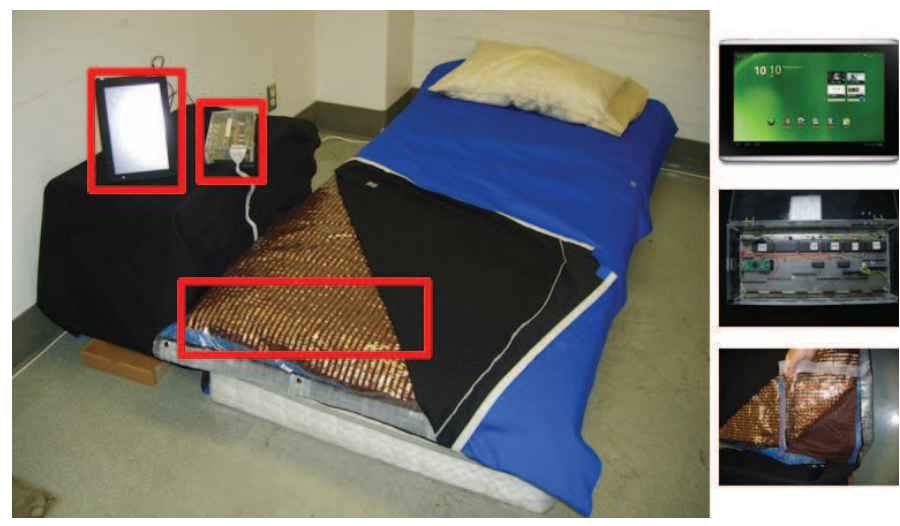

Figure 1. The pressure sensitive bedsheet consists of three parts: a textile sensor sheet, a sampling unit and a tablet.

Figure 1 shows the prototype of our bedsheet system. The system consists of three components: a $64 \times 128$ pressure sensor array, a data sampling unit, and a tablet for data analysis and storage. The sensor array is based on eTextile material which is a fiber-based yarn coated with piezoelectric polymer [16]. The initial resistance between the top and bottom surfaces is high. When extra force is applied on the surface of the eTextile, the intra-fibers will be squeezed together and the electrical resistance will become smaller.

The textile sensor array has a three-layer sandwiched structure: the top layer is normal fabric uniformly coated with 64 parallel conductive lines; an eTextile layer in the middle; and a bottom layer with 128 conductive lines (perpendicular to the top 64 lines). Within this structure, each intersection of conductive lines becomes a pressure sensitive resistor. In total there are effectively 8192 pressure sensors. 


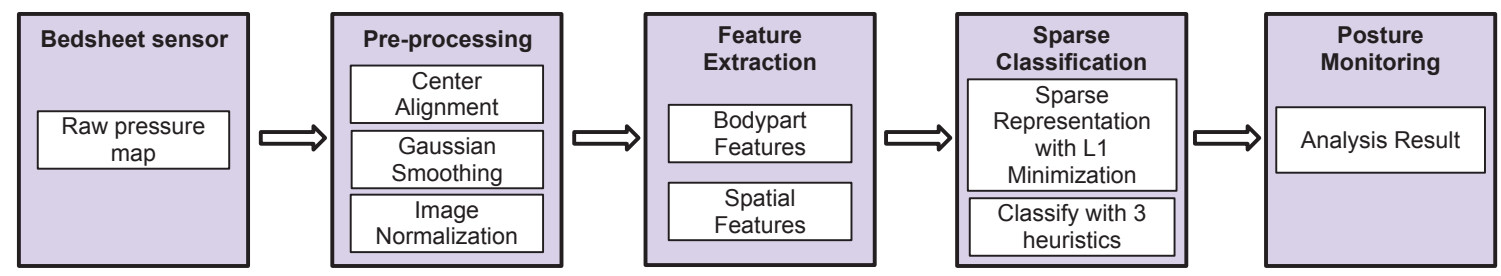

Figure 2. Sleep Posture Recognition Framework

For the scanning, each conductive bus on the bottom is connected to an ADC via analog switch module, S1, and to ground via an offset resistor; each conductive bus on the top is connected to the voltage supply Vcc via analog switch model, S2. The scanning sequence of S1 and S2 is synchronized by a microcontroller. For example, when S2 connects bus $\mathrm{i}$ on the top layer to Vcc and $\mathrm{S} 1$ connects bus $\mathrm{j}$ to ADC, the scan will read the sensor located in row $\mathrm{i}$ and column $\mathrm{j}$. Therefore, this peripheral circuitry has random access to any sensor in the bedsheet. The advantage of this design is the $64 \times 128$ sensor structure has only $192 \mathrm{I} / \mathrm{O}$ pins, one for each of the bus lines. Further details of this scanning circuit design are described in our related work [16].

Figure 3(a) shows an example of a user lying on the bedsheet. The subject sleeps on the bedsheet in a right fetus posture, and the corresponding pressure image is illustrated in Figure 3(b). We can see that body parts (such as hip, legs) are shown clearly in the pressure image due to the dense sensors in the bedsheet. It is helpful to characterize the geometrical features of sleep postures and posture classification, which will be discussed in detail in the next section.

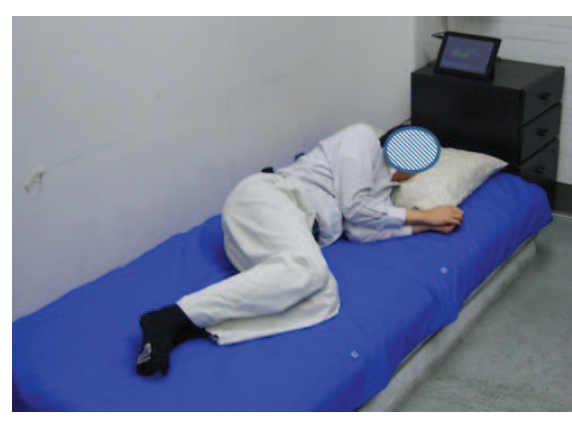

(a) One subject on the bedsheet system

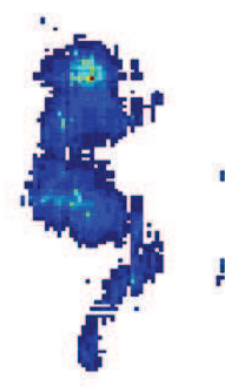

(b) Pressure image
Figure 3. System demonstration with one subject sleeping in a right fetus posture.

\section{Framework For Sleep Posture Analysis}

Figure 2 shows the sleep posture analysis process. The central three steps, Pre-processing, Feature Extraction, and Sparse Classification, will be discussed in this section.

\section{A. Pre-processing}

The pre-processing on the raw pressure images is required so that the images can be standardized in such a way to enable successful classification. The raw images contain noise and artifacts that affect classification, and pre-processing mitigates the side effects as much as possible.

- Firstly, the subject can be located anywhere on the bedsheet, so to correct this, the images are aligned to a common center of mass and relocated to the center of the image.

- A smoothing filter of a symmetric $5 \times 5$ unit normal distribution is applied. This smoothing minimizes the effect of noise in the pressure map.

- The images are rotated so that the dominant axis of the body shape is aligned vertically in the image. The dominant axis is found by an eigenvector calculation by approximating the human body geometry as an ellipse. This accounts for the different lying angles for the subjects.

- The images are normalized so that the sum of pixel weights is one. This step attempts to counteract the affects for the different body mass of patients.

\section{B. Feature Extraction}

Traditional feature extraction methods on images include dimension reduction techniques. Widely popular is Principal Component Analysis [17] which relies on finding the dominant orthogonal axes which maximizes the statistical variances in the data. PCA is largely data dependent and is a general method to find macro structure in datasets. This method has been applied to sleep posture recognition in current literature [18].

In this work, we propose a different method of feature extraction for posture classification that is based on the geometry of the pressure images. It is more attuned to the physical characteristics of the body shape and has a definite physical meaning. An advantage of using these proposed features over PCA reduction is the processing time required to extract these features; our proposed features are based on simple geometry.

In all, we propose 32 features to be extracted from each of the pressure images. The features are described as either 
Spatial features or Bodypart features. Spatial features are those features that describe global aspects of the image such as the proportion of the image that is covered by the subject, how symmetric is the image, and direction of any curvature in the pressure image. Bodypart features are localized features that describe location and size of expected body parts such as the hip and shoulder.

Table I

Global Spatial Features

\begin{tabular}{|c|c|c|}
\hline No. & Name & Description \\
\hline 1 & Coverage & Proportion of image covered \\
\hline 2 & Per25 & Coverage of $25 \%$ of pressure \\
\hline 3 & Per50 & Coverage of 50\% of pressure \\
\hline 4 & Per75 & Coverage of 75\% of pressure \\
\hline $5-12$ & Reg1 - Reg8 & Coverage over 8 fixed rectangular regions \\
\hline 13 & Symmetry & Measure of pressure symmetry \\
\hline 14 & Balance & Measure of pressure on both sides of image \\
\hline 15 & DirCurve & Measure of curvature of pressure image \\
\hline
\end{tabular}

Refer to Table I for a full a listing of the global Spatial features and Table II for the localized Bodypart features. A more detailed explanation of the features follows here. Unless otherwise stated, we will assume the $\mathrm{x}$ axis is along the short side of the bedsheet, the $y$ axis runs along the long side of the bedsheet.

\section{Coverage Features (1-12)}

The first feature is Coverage which is the number of pixels that have non-negative sensor values divided by the total number of pixels. The next 3 features only consider coverage for the pixels that contain $25 \%, 50 \%, 75 \%$ of the total pressure. Features 5-12 are coverage by regions. The regions are 8 equally sized subdivisions of the image as shown in Figure 4. Given that the original dimensions of the image are $64 \times 128$ pixels, the region sizes are $32 \times 32$ pixels.

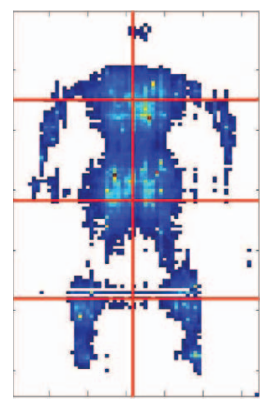

Figure 4. Coverage by Regions: Features 5-12

Symmetry and Balance $(13,14)$

Symmetry is the sum of the absolute value of the difference of pixels on either side of the center image line. A supine posture would have more symmetry than a side posture. Balance is the sum of the difference of pixels on either side of the center image line. This is different to the Symmetry measure; we do not take the absolute value of the difference of pixels. The resulting measure describes which side of the image contains most of the pressure.

\section{Direction of Curvature (15)}

This measure detects the dominant direction of curvature of the body image. A person lying on one side will have a detected body curvature, whereas a supine position should exhibit a straighter pressure image. The steps to extract this feature metric are as follows:

- Create a binary image that contain pixels that are above a suitable threshold. The choice of threshold is obtained experimentally, although a reasonable estimate is $50 \%$ of the peak sensor value.

- Skeletonize the binary image by finding midpoints of boundary pixels (see Figure 5).

- Remove joint pixels from the skeleton so that each curve is separated. Remove curves that are shorter than 5 pixels.

- For all pixels along the curve, find the angle bisector. The director of curvature is taken as the sum of the y components of the angle bisectors, i.e. in the lateral axis of the bedsheet.

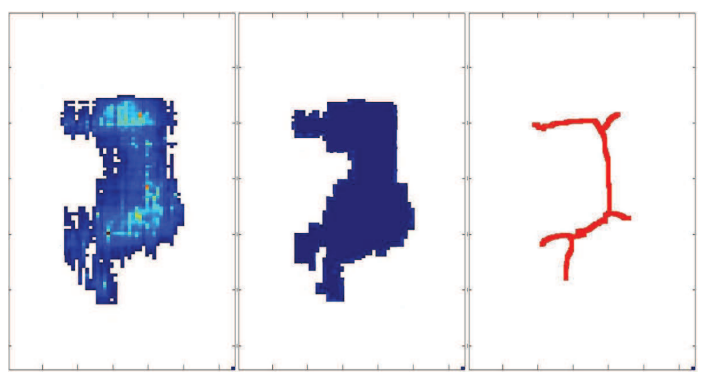

Figure 5. Direction of Curvature feature: Left: original image. Middle: thresholded image. Right: skeletonized image.

Table II

Local GeOMETRICAL Bodypart Features

\begin{tabular}{|c|c|c|}
\hline No. & Name & Description \\
\hline 16,17 & HipPoint & (x, y) location of hip location \\
\hline $18-21$ & HipBox & (x, y, width, height) of bounding box of hip \\
\hline 22 & HipArea & Area in pixels of bounding box of hip \\
\hline 23 & HipPtoBox & Ratio of hip location to bounding box width \\
\hline 24,25 & ShPoint & (x, y) location of shoulder location \\
\hline $26-29$ & ShBox & (x,y,width,height) of bounding box of shoulder \\
\hline 30 & ShArea & Area in pixels of bounding box of shoulder \\
\hline 31 & ShPtoBox & Ratio of shoulder location to box width \\
\hline 32 & HipShDist & Hip to Shoulder Distance \\
\hline
\end{tabular}

Hip Features (16-23)

Since pre-processing of the image is done initially, we make the assumption that the hip is located in the quarter of the image below the center of mass of the pressure image. An estimate of the hip location is taken to be the pixel that 
is located at the weighted center of pixels in this quarter image. The bounding box around the hip is the rectangular region of the pixels that contain $75 \%$ of the pressure value within the quarter image below the center of mass of the pressure image. The ratio of hip location to bounding box width provides a measure that shows where the hip location is in relation to the bounding box of the hip.

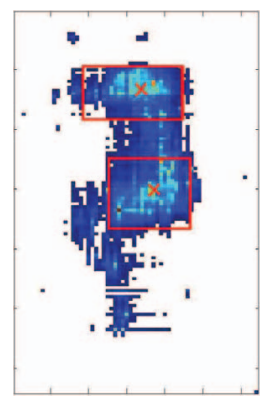

Figure 6. Hip and Shoulder Features

\section{Shoulder Features (24-31)}

Similar to the hip features (16-23) above, we extract the same information for the shoulder. A similar assumption applies to the quarter image above the center of mass for the shoulder location. Figure 6 shows a sample of the locations and bounding boxes of the hip and shoulders. Finally, Feature 32 is the pixel distance between hip and shoulder locations.

Since all the features described above are on different scales, the feature values are scaled and shifted so that the resulting values are in the range $[0,+1]$.

\section{Classification using Sparse Representation}

Sparse Classification has been used previously in other medical analysis and has been shown to have effective performance over a wide range of applications [19]. The classification method comes from the theory of Compressed Sensing [20] which proposes that data exhibits sparsity in some transformed representations. That is, a signal can be represented as a sparse signal in a transformed feature space, and can be accurately re-constructed with a lower sampling rate than the Nyquist-Shannon rate.

Given a data set of $n$ samples, with each sample having $m$ dimensions (features), define the data matrix $A \in R^{m \times n}$ that comprises of these $m$-element column vectors arranged side by side. Now given a new sample $y \in R^{m}$, can a solution $x \in R^{n}$ be found such that $x$ is described in terms of the data set? i.e. can we find $x$ that satisfies:

$$
y=A x .
$$

So $y$ is a linear combination of the columns in the data set, and $x=\left[x_{1}, x_{2}, \ldots, x_{n}\right]^{T}$ is an unknown vector of coefficients. This linear system is underdetermined when there are more unknowns than equations, and hence there are infinitely many solutions for $x$. This is the case for our formulation of posture classification in this paper. However, if certain constraints are imposed, then a unique solution for $x$ will exists that will accurately represent the original sample. There are 3 main sparsity constraints on $x$ that have been considered in literature.

- $l_{0}$ sparsity is defined to minimize the number of nonzero elements of $x$. Solving for $x$ has been shown to be NP-hard [21].

- $l_{2}$ sparsity is the efficient least squares solution, however this is not always equivalent to the $l_{0}$ solution.

- $l_{1}$ sparsity is defined as the minimal sum of absolute values of elements of $x$. Candes et al. [20] have proved that $l_{1}$ sparsity is equivalent to $l_{0}$ and, moreover, can be solved as a convex optimization problem:

$$
\hat{x}=\arg \min _{x}\|x\|_{1},
$$

$$
\text { s.t. } y=A x \text {. }
$$

The sparse representation of a sample is used in classification by matching this representation to a set of class labels. The data set $A$ is composed of training samples and each sample has been assigned a class label, $C_{n}$. Let each training sample be represented as a column vector, $a_{i j}$, where $j$ is the sample number within class $i$. The number of samples need not be the same for each class. The data matrix $A$ is shown here with samples grouped together in their classes:

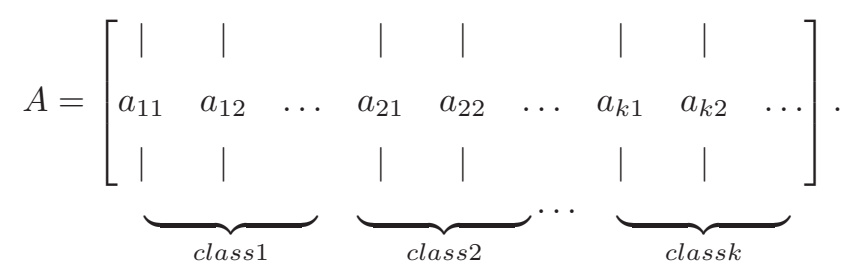

Any test sample $y$ is represented by a linear combination of the training samples:

$$
y=a_{11} x_{1}+a_{12} x_{2}+\cdots+a_{k j} x_{n},
$$

where $k$ is the number of class labels and $j$ is the number of training samples for the $k$-th class.

The $l_{0}$ minimized sparse solution for $x$ will have only a small number of non-zero elements. The training samples that correspond to the non-zero elements are those that can represent the new sample well. We propose 3 heuristics to select the class label given a sparse solution for $x$ and the data set with training labels as follows.

- Maximum Coefficient (MC). The class label belonging to the training sample that corresponds to the largest coefficient of the sparse solution of $x$ is the predicted class label:

$$
\hat{k}=C_{\text {arg } \max _{i}\left(x_{i}\right)} .
$$


- Maximum Sum of Class Coefficients (MSCC). The predicted class label is the class whose sum of coefficients of $x$ is maximized:

$$
\hat{k}=\arg \max _{k}\left(\sum_{i \in a_{k i}} x_{i}\right) .
$$

In other words, for each class $k$, take the sum of the coefficients of $x$ that correspond to the training samples belonging to that class. The predicted label is the class that maximizes these sums. The training samples that are most closely represented to the test sample should correspond to the bulk of elements of the sparse solution to $x$.

- Minimum Class Residual (MCR). An alternate choice for a heuristic to predict the class label is to find the class that minimizes the class residual. The residual is the error between the test sample and the reconstructed sample based on the sparse solution to $x$ :

$$
\text { residual }=\|y-A \hat{x}\|_{2} .
$$

So the predicted class is

$$
\hat{k}=\arg \min _{k}\left\|y-A_{k i} x_{i}\right\|_{2} .
$$

Each of these 3 heuristics are evaluated in the following section.

\section{EXPERIMENTAL RESUlts}

\section{A. Experimental Setup}

We run a pilot study in the lab to evaluate the performance of the system for sleep posture monitoring. There are 14 subjects in the experiment, where 9 subjects are male and 5 subjects are female. The weight of the subjects ranges from $50 \mathrm{~kg}$ to $85 \mathrm{~kg}$, and height between $155 \mathrm{~cm}$ and $188 \mathrm{~cm}$. For the sake of consistency, the bedsheet system is also deployed on a standard twin-size coil spring mattress during the experiments (See Figure 1).

In Idzikowski's study of 1000 people [22], left and right fetus postures, i.e. with legs bent, are most common at $41 \%$. The other side lying postures, i.e. with straight legs, account for $28 \%$ of positions. We refer to these as $\log$ postures. Supine (lying on back) at $8 \%$ and prone (lying on front) at $7 \%$ are the next most common postures. Therefore, for the experimental evaluation, we investigate the 6 postures including Left-Log (LL), Left-Fetus (LF), Right-Log (RL), Right-Fetus (RF), Prone (P) and Supine (S). The examples of these postures are shown in Figure 7.

In the data collection, 40 samples were recorded for each of the 6 postures for each subject. At fixed intervals, the pressure image of the subject's posture was recorded while the subject maintained a comfortable sleeping position. Variations in body, arm and leg positions were allowed and the system is tested on a range of positions that fall within the 6 defined postures. All postures include a standard
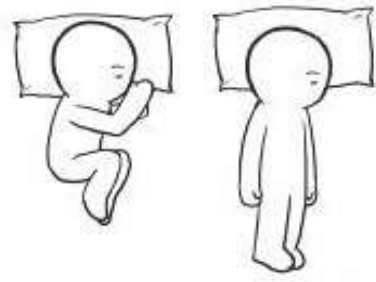

(a) Right Foetus

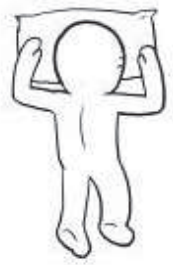

(b) Right Log

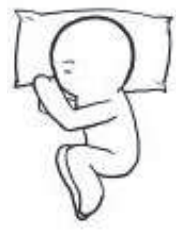

(d) Prone

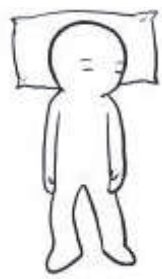

(c) Supine

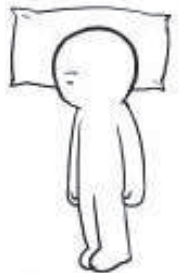

(f) Left $\log$
Figure 7. Six postures used in experiments

queen size pillow for the head. Testing was carried out with Leave One Out Cross Validation by subject, i.e. test on one subject's data with the training data from all the other subjects. Repeat this for each subject. Sparse classifiers are implemented using the CVX convex optimization package [23].

\section{B. Sparse Classification Results}

Table III summarizes the precision and recall results of 6 posture classification using the set of geometric features with different classifiers. The Sparse Classifiers with Maximum Sum of Class Coefficients (MSCC) heuristic and Minimum Class Residual (MCR) heuristics show a good improvement in accuracy over Decision Tree and Nearest Neighbor classifiers.

Table III

COMPARISON TO OTHER CLASSIFIERS

\begin{tabular}{|c|c|c|c|}
\hline & Precision & Recall & Average* \\
\hline C4.5 Decision Tree & $57.0 \%$ & $56.8 \%$ & $56.9 \%$ \\
k-Nearest Neighbor & $64.7 \%$ & $62.1 \%$ & $63.4 \%$ \\
Sparse Classifier (MC) & $\mathbf{6 5 . 4 \%}$ & $\mathbf{6 1 . 0 \%}$ & $\mathbf{6 3 . 2 \%}$ \\
Sparse Classifier (MSCC) & $\mathbf{8 3 . 1 \%}$ & $\mathbf{8 2 . 7 \%}$ & $\mathbf{8 2 . 9 \%}$ \\
Sparse Classifier (MCR) & $\mathbf{8 3 . 5 \%}$ & $\mathbf{8 2 . 9 \%}$ & $\mathbf{8 3 . 2 \%}$ \\
\hline
\end{tabular}

*Average is the arithmetic mean of precision and recall

We note that the Maximum Coefficient (MC) heuristic does not show any improvement in the accuracy over Nearest Neighbor. The reason is that after the transformation into the sparse domain, the Maximum Coefficient is a metric most similar in nature to Nearest Neighbor since it essentially finds the training sample that maximizes its representativeness to the test sample, regardless of the class membership.

We look more closely at the results of the Spare Classifiers and note the confusion matrices given in Tables IV to VII. 
Table IV

CONFUSION MATRIX NEAREST NEIGHBOR

\begin{tabular}{|c|c|c|c|c|c|c|c|}
\hline & LL & LF & P & RL & RF & S & Recall \\
\hline LL & 123 & 6 & 7 & 26 & 41 & 83 & $43 \%$ \\
LF & 3 & 194 & 14 & 39 & 3 & 33 & $68 \%$ \\
P & 1 & 27 & 234 & 0 & 19 & 5 & $82 \%$ \\
RL & 46 & 21 & 0 & 171 & 6 & 42 & $60 \%$ \\
RF & 45 & 9 & 14 & 25 & 185 & 12 & $64 \%$ \\
S & 42 & 6 & 3 & 20 & 5 & 210 & $73 \%$ \\
\hline Precision & $47 \%$ & $74 \%$ & $86 \%$ & $61 \%$ & $71 \%$ & $55 \%$ & \\
\hline
\end{tabular}

Table V

CONFusion Matrix SPARse Classifier (MC)

\begin{tabular}{|c|c|c|c|c|c|c|c|}
\hline & LL & LF & P & RL & RF & S & Recall \\
\hline LL & 149 & 29 & 24 & 19 & 23 & 42 & $52 \%$ \\
LF & 15 & 168 & 26 & 30 & 18 & 29 & $59 \%$ \\
P & 9 & 24 & 202 & 6 & 20 & 25 & $71 \%$ \\
RL & 40 & 25 & 5 & 176 & 22 & 18 & $61 \%$ \\
RF & 11 & 22 & 23 & 19 & 194 & 21 & $67 \%$ \\
S & 44 & 13 & 11 & 9 & 11 & 198 & $69 \%$ \\
\hline Precision & $56 \%$ & $60 \%$ & $69 \%$ & $68 \%$ & $67 \%$ & $59 \%$ & \\
\hline
\end{tabular}

Table VI

Confusion Matrix Sparse Classifier (MSCC)

\begin{tabular}{|c|c|c|c|c|c|c|c|}
\hline & LL & LF & P & RL & RF & S & Recall \\
\hline LL & 207 & 6 & 2 & 15 & 6 & 50 & $72 \%$ \\
LF & 4 & 249 & 14 & 6 & 9 & 4 & $87 \%$ \\
P & 1 & 22 & 245 & 1 & 12 & 5 & $86 \%$ \\
RL & 14 & 27 & 1 & 219 & 12 & 13 & $77 \%$ \\
RF & 0 & 9 & 14 & 3 & 262 & 2 & $90 \%$ \\
S & 30 & 6 & 2 & 1 & 3 & 244 & $85 \%$ \\
\hline Precision & $81 \%$ & $78 \%$ & $88 \%$ & $89 \%$ & $86 \%$ & $77 \%$ & \\
\hline
\end{tabular}

Table VII

CONFUSION MATRIX SPARSE Classifier (MCR)

\begin{tabular}{|c|c|c|c|c|c|c|c|}
\hline & LL & LF & P & RL & RF & S & Recall \\
\hline LL & 202 & 8 & 5 & 18 & 5 & 48 & $71 \%$ \\
LF & 2 & 252 & 18 & 5 & 6 & 3 & $88 \%$ \\
P & 1 & 22 & 249 & 1 & 10 & 3 & $87 \%$ \\
RL & 12 & 28 & 2 & 225 & 10 & 9 & $79 \%$ \\
RF & 0 & 11 & 15 & 5 & 257 & 2 & $89 \%$ \\
S & 27 & 9 & 3 & 1 & 3 & 243 & $85 \%$ \\
\hline Precision & $82 \%$ & $76 \%$ & $85 \%$ & $88 \%$ & $88 \%$ & $79 \%$ & \\
\hline
\end{tabular}

Table IV shows the confusion matrix for Nearest Neighbor classifier and we note the similarity with the Maximum Coefficient Sparse Classifier (MC) in Table V.

Generally, the log postures are harder to recognize than the other postures. Recall for both Left Log and Right Log are always lower than for the other 4 postures, while the precision rates are generally lower but not always. The log postures are most similar to each other since both have legs outstretched and arm positions can vary. The next most similar posture is Supine. This is seen in all of the confusion matrices as high counts for predicted switched Left Log and Right Log, and Supine.

Figure 8 shows two examples of misclassifications of hard log postures. The left image shows Left Log posture

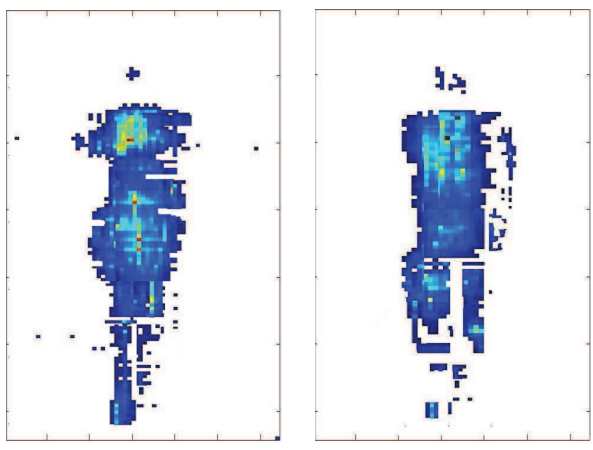

Figure 8. Misclassified postures. Left image is a Left Log posture that is classified as Right Log. Right image is a Right Log posture that is classified as Supine.

that is incorrectly identified as Right-Log. This typical kind of error can be explained from the pressure map that is extended behind the subject's back. Hence misclassifications can occur since the pressure image now looks like a Right Log image. Similarly, the right image shows Right Log posture that is incorrectly identified this time as Supine. Visually, this image is hard to not identify as Supine.

Personalized training can further enhance the classification accuracy of this system. A generalized system that will be pre-trained on a large cohort of trial subjects will also enable improved accuracy. Such a system would be easy to use and not rely on patient specific setup.

\section{Stability Analysis}

We also analyse the robustness of the classification by investigating the variation during cross validation. Stability is a measure that describes how closely the classifier evaluates results if given different data. Figure 9 shows the classification variation for the 6 postures for each of the classifiers.

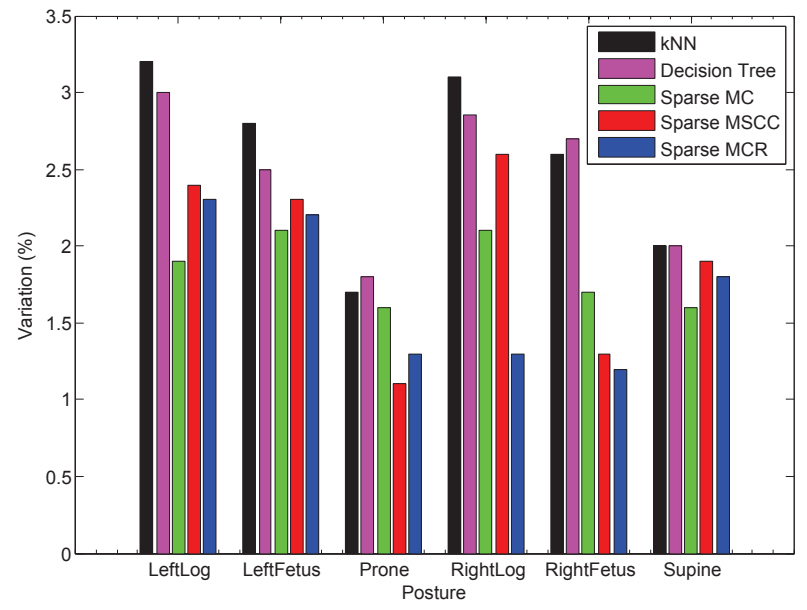

Figure 9. Classification variation for 6 postures 
The Sparse Classifiers have smaller variation values than the other traditional classifiers. This indicates that sparse classification is a more stable classification method. The log postures have a higher variation in classification accuracy than the other postures. This is explained by the higher variation in weight distribution for these postures. In our data, the log postures vary much between fully lying on the side and lying on the back.

Although the Maximum Coefficient (MC) Sparse Classifier achieves the same accuracy as Nearest Neighbor, it does perform better when considering its better stability.

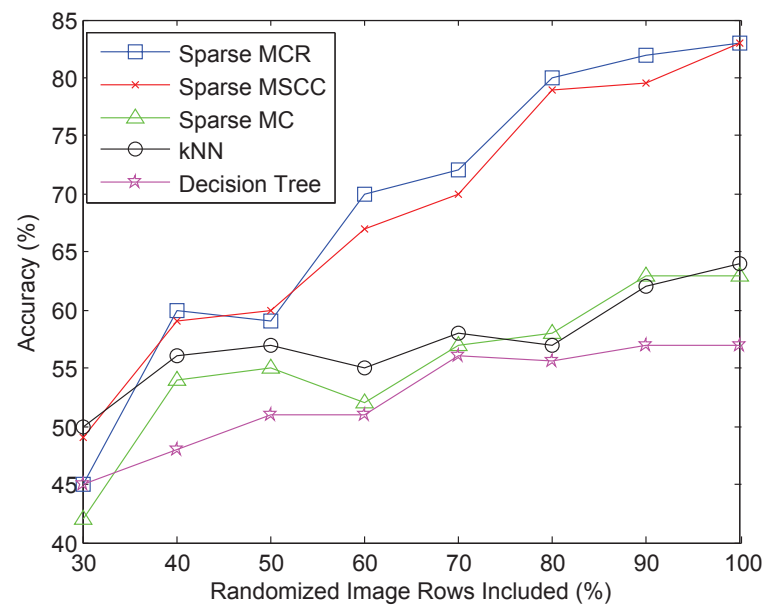

Figure 10. Classification robustness with random row deletions

We also consider the robustness of the algorithm with regards to input errors, such as from disconnected wires in the bedsheet. The effect of this would be missing rows or columns of data in the pressure image. Figure 10 shows experimentally how a random number of failures of bus lines will affect the accuracy of the classification result. Roughly $20 \%$ of the hardware connections can fail with only a $4 \%$ drop in classification accuracy.

\section{Feature Selection}

In this section, we examine the effect of feature selection in classification. 32 features are extracted from the pressure image set for classification. It is most certainly the case that some features are redundant or do not have any effect on classification results. We employ Sequential Forward Selection (SFS) to find subsets of features that are most descriptive of the whole feature set [24]. This method is considered a wrapper method, ie the feature selection is based on using the classification results themselves and the selection process wraps around the classification.

With this method, the first feature is selected by testing each feature individually in classification. The feature with the highest accuracy performance is chosen first. In the second round of selection, each of the remaining features

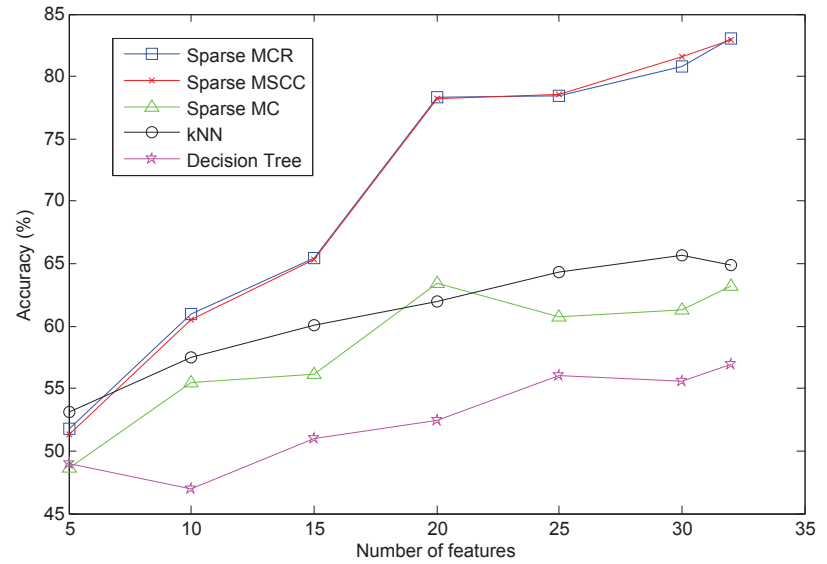

Figure 11. The impact of feature dimension on classification accuracy based on Sequential Feature Selection

is used with the first feature in classification. The feature with the highest accuracy result is chosen as the second feature in the feature selected subset. This process continues as one feature is added at a time until all of the features are selected. Since the classification always uses previously selected features, redundant features are not selected until the end.

Figure 11 shows the relationship between number of selected features chosen using SFS and classification accuracy for each of the classifiers. Accuracy generally increases as more features are used for all of the classifiers. There is a sharp increase in accuracy using the Sparse Classifier with both Maximum Sum of Class Coefficients (MSCC) and Minimum Class Residual (MCR) heuristics, from $65 \%$ to $78 \%$ between 15 features and 20 features. Moreover the three Sparse Classifiers appear to have a threshold at 20 features in the accuracy results, with a smaller rate of increase in accuracy as more features are used. The traditional kNN and Decision Tree show modest increase of about $10 \%$ throughout the whole feature selection process.

\section{CONCLUSION}

This work presents a sleep analysis design that monitors sleep posture using a pressure sensitive bedsheet. An application for such a system is to enable caregivers the ability to automatically identify when patients are at risk of developing pressure ulcers or when subjects experience sleep apnea. This work also presents the novel use of relevant features that can be extracted from pressure images, as well as state of the art classification methods. We developed three heuristics for sparse classification, and in our experiments, show that both Maximum Sum of Class Coefficients (MSCC) and Minimum Class Residual (MCR) heuristics produce reliable sleep posture estimation.

Pressure monitoring systems need not be limited to sleep posture recognition. In nursing home settings, evaluation of 
fall risk is a desirable endeavor. Advanced beds are now being developed that can re-distribute support to different regions of the bed [25] and also aid in the heat flow through the bed mattress. Using pressure point monitoring, the goal is to increase healing speed of patients.

Future work also involves the ability to monitor transitional states as patients move between pre-defined stable classified postures. There are more challenges here because of the large variations in different subjects' motions. 3D model reconstruction of patients from $2 \mathrm{D}$ pressure image is another goal that can be accomplished using the results of this research work.

\section{ACKNOWLEDGMENT}

The authors would like to thank Medisens Wireless Inc. for their insights and building the hardware.

\section{REFERENCES}

[1] J. Parish, "Sleep-related problems in common medical conditions," Chest Journal, vol. 135, no. 2, pp. 563-572, 2009.

[2] M. Thase, "Depression and sleep: Pathophysiology and treatment," Dialogues in Clinical Neurosciences, vol. 135, no. 2, pp. 217-226, 2006.

[3] J. B. Lee, Y. H. Park, J. H. Hong, S. H. Lee, K. H. Jung, J. H. Kim, H. Yi, and C. Shin, "Determining optimal sleep position in patients with positional sleep-disordered breathing using response surface analysis," Journal of Sleep Research, vol. 18 , no. 1 , pp. 26-35, 2009.

[4] C. Ambrogio, X. Lowman, M. Kuo, J. Malo, A. Prasad, and S. Parthasarathy, "Sleep and non-invasive ventilation in patients with chronic respiratory insufficiency," Intensive Care Medicine, vol. 35, pp. 306-313.

[5] A. Oksenberg and D. Silverberg, "The effect of body posture on sleep-related breathing disorders: Facts and therapeutic implications," Sleep Medicine Reviews, vol. 2, no. 3, pp. 139162,2006

[6] C. M. Shapiro, G. M. Devins, and M. R. Hussain, "Abc of sleep disorders. sleep problems in patients with medical illness." BMJ, vol. 306, no. 6891, pp. 1532-1535, 61993.

[7] T. Perneger, C. Heliot, A.-C. Rae, F. Borst, and J.-M. Gaspoz, "Hospital-acquired pressure ulcers: Risk factors and use of preventive devices," Archives of Internal Medicine, vol. 158, no. 17, pp. 1940-1945, 1998.

[8] K. Nakajima, Y. Matsumoto, and T. Tamura, "A monitor for posture changes and respiration in bed using real time image sequence analysis," in Engineering in Medicine and Biology Society, 2000. Proc. 22nd IEEE, vol. 1, pp. 51-54.

[9] W.-H. Liao and C.-M. Yang, "Video-based activity and movement pattern analysis in overnight sleep studies," in Pattern Recognition, 2008. ICPR 2008. 19th International Conference, Dec. 2008, pp. 1-4.
[10] A. Sadeh and C. Acebo, "The role of actigraphy in sleep medicine," Sleep Medicine Reviews, vol. 135, no. 2, pp. 217226, 2002.

[11] Y. Kishimoto, A. Akahori, and K. Oguri, "Estimation of sleeping posture for $\mathrm{m}$-health by a wearable tri-axis accelerometer," in Medical Devices and Biosensors. 3rd IEEE/EMBS International Summer School, Sept. 2006, pp. $45-48$.

[12] E. Hoque, R. F. Dickerson, and J. A. Stankovic, "Monitoring body positions and movements during sleep using wisps," in Wireless Health 2010, ser. WH '10, 2010, pp. 44-53.

[13] M. Jones, R. Goubran, and F. Knoefel, "Identifying movement onset times for a bed-based pressure sensor array," in Medical Measurement and Applications. MeMea IEEE International Workshop, April 2006, pp. 111-114.

[14] Xsensor, http://www.xsensor.com.

[15] VistaMedical, http://www.pressuremapping.com.

[16] W. Xu, Z. Li, M.-C. Huang, N. Amini, and M. Sarrafzadeh, "eCushion: An eTextile Device for Sitting Posture Monitoring," in Body Sensor Networks (BSN), May 2011, pp. 194 199.

[17] I. Jolliffe, Principal Component Analysis. Springer Verlag, 1986.

[18] R. Yousefi, S. Ostadabbas, M. Faezipour, M. Farshbaf, M. Nourani, L. Tamil, and M. Pompeo, "Bed posture classification for pressure ulcer prevention," in Engineering in Medicine and Biology Society, Sept. 2011, pp. 7175-7178.

[19] W. Xu, M. Zhang, A. Sawchuk, and M. Sarrafzadeh, "Corecognition of human activity and sensor location via compressed sensing in wearable body sensor networks," in Wearable and Implantable Body Sensor Networks (BSN), May 2012, pp. 124-129.

[20] E. Candes, J. Romberg, and T. Tao, "Robust uncertainty principles: exact signal reconstruction from highly incomplete frequency information," Information Theory, IEEE Transactions, vol. 52, no. 2, pp. 489-509, Feb. 2006.

[21] B. K. Natarajan, "Sparse approximate solutions to linear systems," SIAM J. Comput., vol. 24, no. 2, pp. 227-234, Apr. 1995.

[22] C. Idzikowski, Beating Insomnia: How to Get a Good Night's Sleep. Gill \& MacMillan, 2003.

[23] CVX Research Inc., "CVX: Matlab software for disciplined convex programming, version 2.0," http://cvxr.com/cvx, Aug. 2012.

[24] G. H. John, R. Kohavi, and K. Pfleger, "Irrelevant features and the subset selection problem," in Machine Learning: Procs. 11th International Conference. Morgan Kaufmann, 1994, pp. 121-129.

[25] Hillrom, http://www.hill-rom.com. 